\title{
Integrated visualization and simulation for lifting operations of modules under congested environment
}

\author{
SangHyeok Han ${ }^{a}$, Zhen Lei ${ }^{a}$, Ahmed Bouferguène ${ }^{b}$, Mohamed Al-Hussein ${ }^{a}$, and Ulrich \\ Hermann ${ }^{c}$
}

${ }^{a}$ Hole School of Construction Engineering, University of Alberta, Canada

${ }^{b}$ Campus Saint-Jean, University of Alberta, Canada

${ }^{c}$ PCL Industrial Management Inc., Canada

Corresponding author email: sanghyeo@ualberta.ca

\begin{abstract}
-
Modular-based heavy construction projects are recognized as faster, safer, and more efficient than traditional those completed through on-site construction methods. The successful completion of these projects relies on an efficient material handling system, especially when mobile cranes are utilized to satisfy heavy lift requirements. However, engineers are faced with the challenge of planning the lifting operations of heavy modules within congested areas. Currently, mobile crane analysis is implemented manually, but requires timeconsuming data input resulting in an increase of errors and a lack of proper crane productivity performance analysis; this complicates the planning process. To overcome these limitations, the research presented in this paper proposes to integrate visualization and simulation, in an approach known as post-3D visualization simulation, in order to plan collision-free crane lifts by eliminating potential errors in 3D visualization and to predict crane productivity performance in simulations. This proposed system will contribute to the successful completion of construction projects with high productivity and site-error reduction by selecting the best crane operation that includes various crane lifts. An actual industrial project which has a number of constraints, including space limitations, different types of site layouts, and various crane lifts, is used to validate the proposed framework.
\end{abstract}

\section{Keywords -}

Mobile crane; post-3D visualization simulation; crane performance; productivity; industrial projects

\section{Introduction}

The Athabasca Oil Sands in Alberta, Canada is becoming one of the most attractive industrial construction markets in the world. Oil sands-related projects are built utilizing the modular-based (off-site) construction method. The successful completion of modular-based industrial construction projects, in terms of saving time and reducing associated costs, relies heavily on a well-designed material handling systems with regards to the operation of construction equipment. Among this construction equipment, mobile cranes are frequently utilized in order to lift modules to their positions for installation since they satisfy the required lift capacity requirements. This frequent utilization of mobile cranes is due to the fact that modules in modular-based industrial construction projects are becoming larger and heavier.

Crane lift studies can be divided into the following categories: (i) crane type selection and location [4, 13, 21, 22, 24, 25]; (ii) crane support system [11]; and (iii) crane lift path planning [1, 3, 7, 17, 19, 23]. When lift studies are implemented, the most critical step in terms of safety and productivity is to identify and eliminate potential collision errors. To facilitate this step efficiently and effectively, many researchers have searched for better algorithms and superior techniques such as simulation, 3D visualization, and integrated systems $[5,14,18,26]$. Corresponding to this trend, the effectiveness of $3 \mathrm{D}$ visualization has been reported in actual cases [9, 19]. Crane productivity analysis which takes into account $\mathrm{CO}_{2}$ emissions has been introduced for utilization of a tower crane on a high-rise building project [11]. However, previous research has not been fully adopted in practice, especially in heavy industrial construction projects where complicated and timeconsuming algorithms are involved. The current mobile crane analysis is implemented manually but requires time-consuming data input, resulting in an increase of errors and a lack of proper crane productivity performance analysis; this leads to complication of the planning process. To overcome this lack of proper crane productivity performance analysis, crane performance should be evaluated among various scenarios of crane operations in order to select the best crane operation. This paper thus proposes to integrate $3 \mathrm{D}$ visualization and simulation in a so-called post-3D visualization 
simulation system. This system is able to design feasible motions of mobile crane operation in 3D visualization, and has the ability to evaluate the associated crane operation automatically in terms of crane performances in the simulation so that lifting engineers and project participants can actively use them to select the best crane operation. A case study constructed by PCL Industrial Management Inc. is used to validate this methodology.

\section{Methodology}

Among various crane types, such as hydraulic truck cranes, all-terrain cranes, rough-terrain cranes, crawler cranes, and carry-deck cranes, crawler cranes equipped with superlift (tail-swing) are selected for this research in order to accommodate the high lift capacity requirements of heavy industrial projects. According to previous research [8], mobile crane operation studies can be divided into two categories corresponding to the type of operation: (i) crane binary checking [14], which involves checking pick from fixed position (PFP) operations to identify the existence of a path and return a binary result (yes/no); and (ii) crane walking path checking, which searches for pick and walking operations (PWOs) to explore feasible walking paths. PWOs involve the crane walking from a given position for loading to a point from which the crane can reach the set positions of the lifted objects when PFPs are not possible. Since modular-based industrial construction sites consist of numerous modules in congested areas, spatial constraints such as the geometry of the mobile crane and existing obstacles (e.g., modules already installed before installation of lifted objects) are the main issues to consider when designing safe and efficient crane operations. Therefore, virtual motion planning of crane operation (VMCO) plans detailed motions of mobile crane operations by detecting and eliminating potential collision errors in order to validate the PFP or PWO for lifted objects. At this juncture, it each lifted object may have more than one possible crane operation. This is due to the fact that crane lift binary checking and crane walking path systems only search for the feasibility of crane operations on numerous locations for each individual lifted object. In analyzing crane performance, crane lift binary checking, crane walking path, and virtual motion planning systems are inadequate. As a result, these systems can lead to difficulty in selecting the best crane operation when the lifted object has more than one possible crane operation. To eliminate this inadequacy in the performance systems, this paper proposes to integrate $3 \mathrm{D}$ visualization and simulation, known as post-3D visualization simulation, in order to predict the crane performance when lifting objects. Industrial projects involve a large number of lifts, thereby challenging the manual system of implementation in lift studies. All the systems in the proposed methodology are therefore executed automatically.

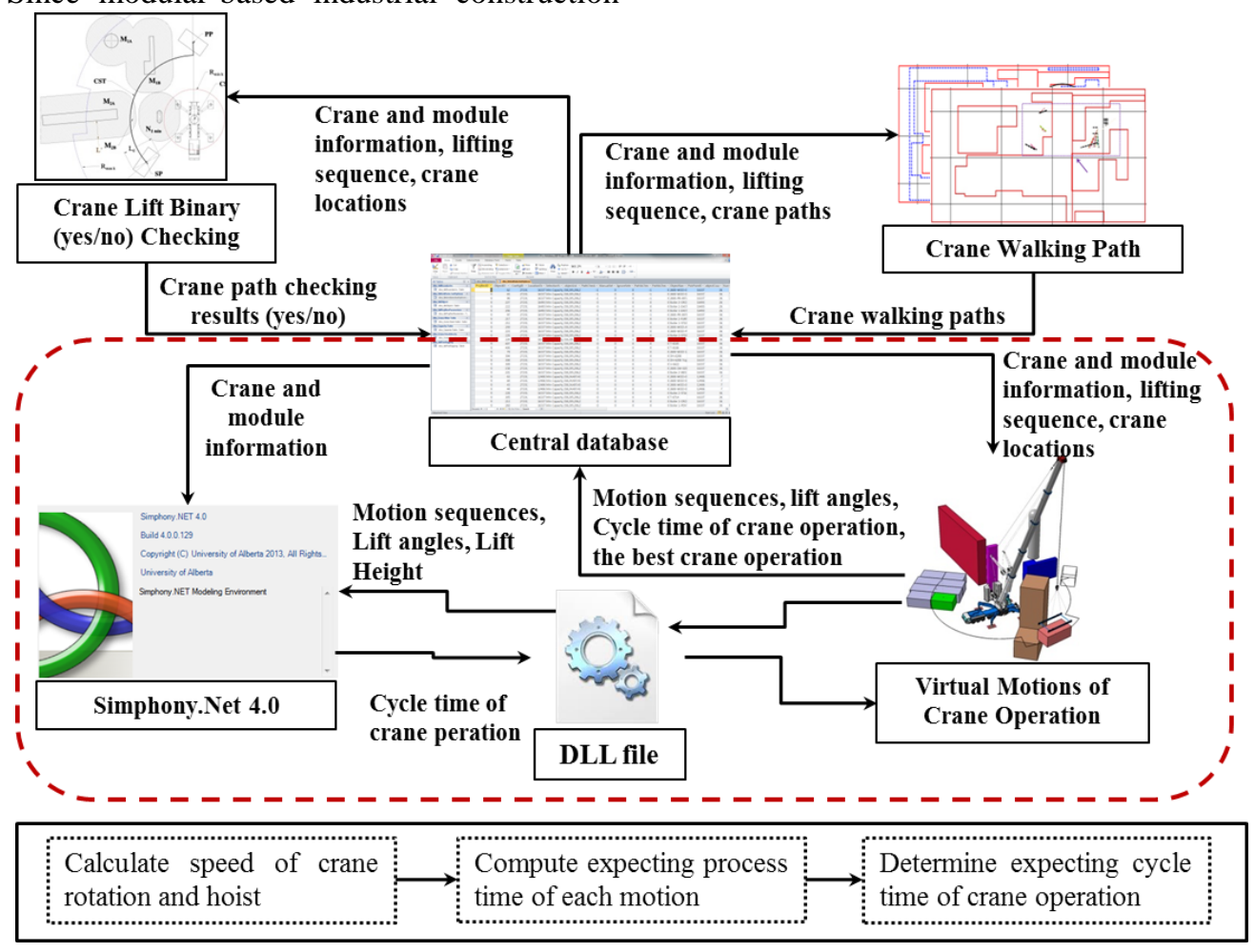


Figure 1. Proposed Methodology

\begin{abstract}
As shown in Figure 1, the simulation is implemented for the evaluation of crane performance after designing detailed motions of mobile crane operations in a $3 \mathrm{D}$ environment. The input data consists of: (i) crane information, including crane configurations, crane speeds, and capacity charts provided by manufacturers; (ii) rigging (e.g., spreader bar, sling, and hook) information, such as total weights based on different module types; (iii) module information, including module weights; and (iv) motion sequences and associated lift angles. The output of the post-3D visualization simulation is the best crane operation in terms of cycle times. The VMCO controller is an execution tool for the post-3D visualization simulation, which is developed using Maxscript, a built-in language tool in 3D Studio (3ds) Max.
\end{abstract}

\subsection{Post-3D Visualization Simulation}

Computer simulation is used to design a mathematical-logical model of a real world system and experiment with the model on a computer. The purpose of using a simulation is to eliminate unforeseen bottlenecks, to use resources ore effectively, and to optimize system performance before an existing system is changed by the proposed design. 3D visualization, meanwhile, is recognized as a comprehensive 3D computer-aided design tool used to design the construction facility and its associated schedule; when these two aspects interact, the workability of a schedule is ensured and alternative construction strategies in terms of spatial and workflow issues are configured. However, each of these systems has their own limitations [10]. To reinforce their functionalities, some researchers have integrated simulation and 3D visualization in a system referred to as post-simulation visualization [10]. This system runs the simulation in order to analyze the productivity of a project, and then identifies spatial issues in order to check workability in 3D visualization. However, this system is not currently used for crane lift studies, which require the analysis of spatial issues in oil sands projects that involve large numbers of lifted objects, complexity, and various site layout changes. Each lifted object also may have more than one feasible crane operation, leading to challenges in selecting the best crane operation. Therefore, this paper proposes a post-3D visualization simulation which plans mobile crane operations in a simulation by calculating the required lift angles of each crane configuration and preventing potential collision errors [8]. It then simulates crane performance which is addressed in this paper as a means to analyze the process times of crane operations for lifted objects.

3ds Max, a modelling tool, and Simphony.NET [6], a simulation tool, are used to demonstrate the effectiveness of the proposed methodology. Previous studies [2, 10] have highlighted four limitations encountered in integrating these tools: (1) lack of information exchange; (2) insufficient automation; (3) only one-way information exchange from simulation to 3ds Max; and (4) inefficient information checking. These limitations result in an ASCII file type as a intermediate step whenever users try to implement information exchange from the simulation to $3 \mathrm{~d}$ s Max. As well, outputs generated from both simulation and visualization tools are not represented in an environment, so this may lead to poorly managed decision making between project participants. To capture evolving technologies, 3ds Max provides better information exchange that connects to external databases such as Microsoft Access using the VMCO controller developed in 3ds Max. Corresponding to this technology, this paper uses a Dynamic Link Library (DLL) file type, described in Figure 2, which provides a library of executable functions that can be used by windows applications to implement information exchange quickly and easily, and to execute functionalities of Simphony.NET in 3ds Max without opening a simulation model to manually input data.

To analyze crane performance for lifted objects, the VMCO controller transfers the following information: (1) module ID; (2) tracking ID; (3) radii during each motion of crane operation; (4) total weight; (5) capacity; (6) factor; (7) motion ID; (8) lift angles; (9) lift height; and (10) speed. Since there is not enough time data (probability data) to implement a simulation model in order to predict mobile crane productivity, this paper uses the simulation as a deterministic model. The VMCO controller determines the process time of each motion of the crane operation, based on the speed and lifting capacity provided by manufacturers and the total weights satisfied in Equation (1). At this juncture, total weight may have variation since the various modules may have differing weights and be loaded by numerous types of spreader bars.

$$
W_{\text {Total }}=W_{\text {Liffed }}+W_{\text {Spreadbar }}+W_{\text {Sling }}+W_{\text {Hook }}
$$

Where $W_{\text {Total }}$ is the total weight; $W_{\text {Lifted }}$ is the weight of the lifted object; $W_{\text {spreaderbar }}$ is the weight of the spreader bar; $W_{\text {Sling }}$ is the weight of the sling; and $W_{\text {Hook }}$ is the weight of the hook.

After the total weight is calculated, the factor is 
calculated by the total weight divided by the crane lift capacity setting identified in the crane lift capacity chart; this capacity is based on the working radius from center position of crane to center position of lifted object for a specific motion in 3D visualization. The factor indicates how much of the crane lift capacity is occupied by the total weight. Using this factor, speed can be also calculated.

Based on site layout and the sequences of crane operation shown in Table 1, the virtual motion planning system plots detailed motions of the mobile crane operations for lifted objects. It should be noted that the sequences of crane operation used in this paper follow practical crane operation, where the crane lifts the object as close as possible to the ground after loading (motion ID 6). The rigging system is lifted up to maintain particular clearances as defined by users (motion ID 13) whenever the distance between the lifted object and existing obstacles on-site is less than or the same as the clearance during the crane operation across animation time. Based on these sequences, a simulation model is built in Simphony.NET 4.0 .

To achieve a post-3D visualization simulation system, the DLL file in 3ds Max is used to integrate the simulation and $3 \mathrm{D}$ visualization with automated information exchange. That is, the VMCO controller delivers the required information to the simulation model through the DLL file developed in Visual Studio.NET, which represents module ID, tracking ID, and cycle time of the mobile crane operation generated from the simulation model. The cycle times are used to identify the best crane operation (i.e., the shortest cycle time) by comparing the cycle times of the crane operations. As a result, the system can assist lift engineers and project managers to implement decision making quickly and easily by selecting the best crane operation. This is achieved by comparing the cycle times of various crane operations, and by designing the lift schedule in a manner which takes into account other material handling systems as well as the entire project schedule.

Table 1. Sequences of crane operation

\begin{tabular}{c|l}
\hline Motion ID & \multicolumn{1}{c}{ Description } \\
\hline \hline 1 & Crane base Rotation \\
2 & Superstructure Rotation \\
3 & Boom Rotation \\
4 & Rigging Rotation \\
5 & Rigging Down for load \\
6 & Rigging up with load \\
7 & Superstructure Rotation with load \\
8 & Crane walking with load \\
9 & Superstructure Rotation with load \\
10 & Boom Rotation with load \\
11 & Rigging Rotation with load \\
12 & Rigging Down with load \\
13 & Rigging Up
\end{tabular}

\section{Implementation}

This paper implements the methodology in a modular-based oil sands project constructed by PCL Industrial Management Inc. in Alberta, Canada. Target areas in the case study have a total of 150 lifting cases. The PFP method is required for 140 cases, while 10 modules are installed by the PWO method. The Demag CC 2800 crawler crane equipped with superlift is selected as the crane type in this case study (Capacity: 660 tons, Boom length: $276 \mathrm{ft}$ ). To clearly illustrate the proposed methodology, this paper focuses on the PWO to deliver module ID 103 (Figure 2 describes the site layout). The VMCO reads information from a central database, based upon which it automatically builds 3D models such as a module, existing obstacles, and inside site boundary limits (ISBLs), which are inaccessible areas for the crane. The dimensions of the module are $6 \mathrm{~m}(19.68 \mathrm{ft})$ $\times 36.6 \mathrm{~m}(120.08 \mathrm{ft}) \times 4.1 \mathrm{~m}(13.45 \mathrm{ft})$, and the two crane walking paths (tracking IDs 12049 and 12068) are found to be available.

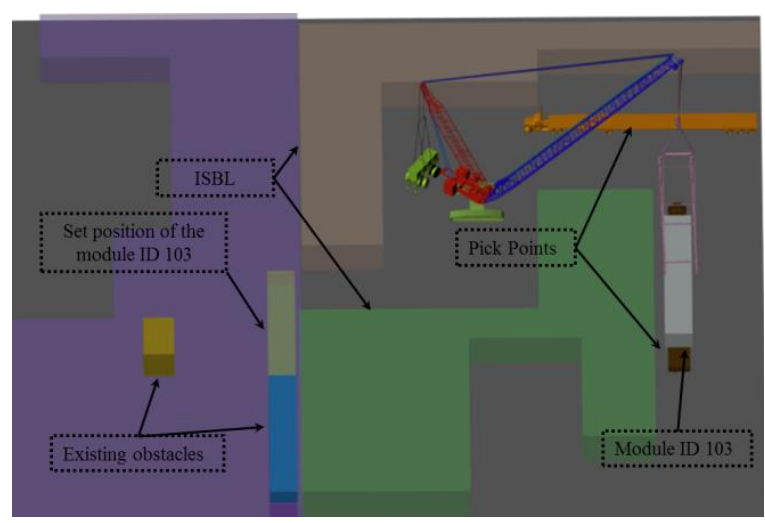

Figure 2. Site layout for module ID 103

Based on two feasible crane walking paths (WPs), including a start point (SP) for loading and a finish point (FP) for unloading, the VMCO controller automatically develops 3D visualization of mobile crane operations by calculating lift angles of each crane configuration and maintaining the clearances defined by users. Figure 3 represents the virtual motions of crane operation for tracking ID 12049 and 12068 in module ID 103. For example, tracking ID 12049, the crane operation to lift the module, is designed based on the following sequence: (1) move and rotate by $73.9^{\circ}$ in the counter-clockwise direction beginning at the SP of the WP; (2) rotate the superstructure by $81.1^{\circ}$ in the clockwise direction to a pick point of the module; (3) rotate the boom upward by $6.8^{\circ}$ to coincide with the location of the rigging system; (4) rotate the rigging system by $98.8^{\circ}$ and lift 
it up and down for loading; (5) rotate the superstructure by $108.4^{\circ}$ to the FP of the WP; (6) walk the crane; and (7) repeat steps (2) to (4) with other variables until the module is installed successfully on its set position. According to this sequence of crane operation, the VMCO controller generates the relevant information (see Table 2): (1) module ID; (2) tracking ID; (3) weight; (4) radius; (5) capacity; (6) factor; (7) motion ID; (8) lift angle; (9) lift height; and (10) various speeds. In the same rule described above, the 3D visualization for the other feasible crane location is developed and relevant information is generated.

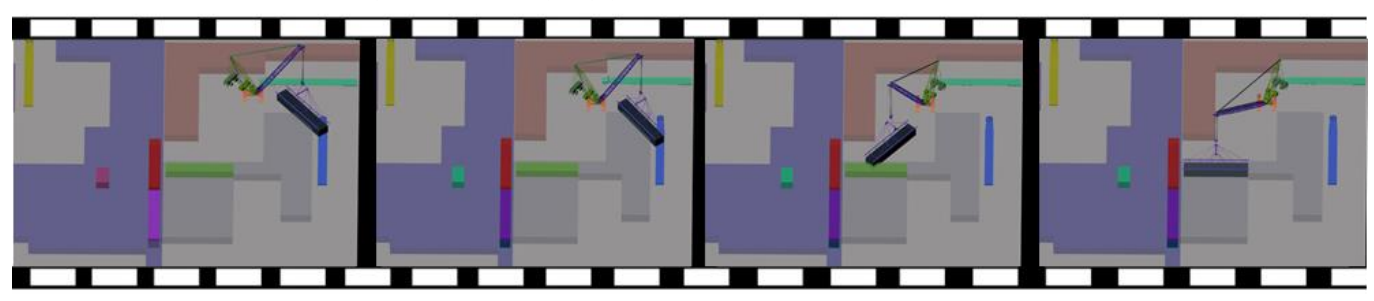

(a) Tracking ID 12049

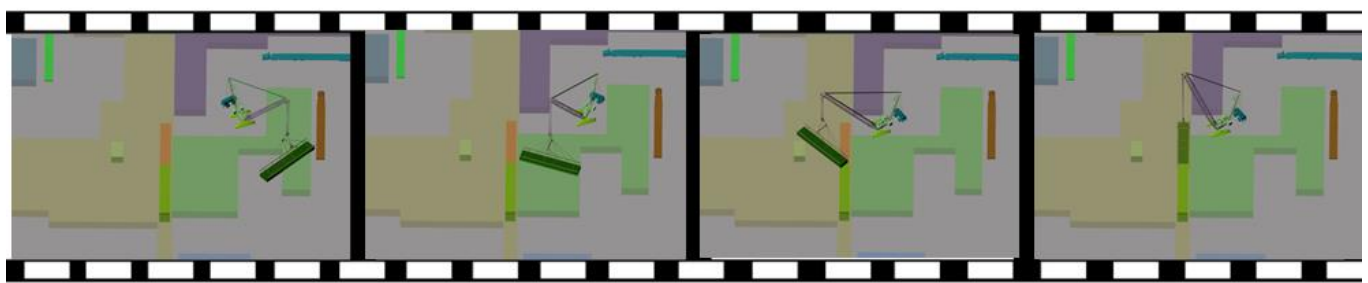

(b) Tracking ID 12068

Figure 3. Crane operations for module ID 103

To evaluate the expected performance of each crane operation, the following information is required for the simulation: (1) module ID; (2) tracking ID; (3) lift angle; (4) lift height; (5) speed; and (6) motion ID. The VMCO controller transfers this information to the simulation model efficiently and effectively through the DLL file. Once the simulation model has run completely, the cycle times of each crane operation (outputs of the simulation) are also shifted to the VMCO controller through the DLL file. This workflow of the automated post-3D visualization simulation system is successful in the information exchange. As a result, the VMCO controller represents the module ID, the tracking ID, and the expected cycle time of each crane operation (Figure 4) in 3ds Max. Based on this information, users can identify which crane WP is the best option to ensure high crane performance and project productivity. Tracking ID 12049 for module ID 103 is found to be the crane location which facilitates the best crane operation, with a cycle time of 11.8253 minutes for the lift.

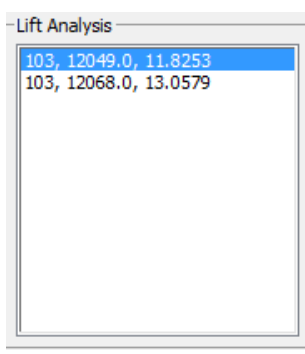

Figure 4. Simulation outputs in VMCO controller

\section{Conclusion}

Industrial construction projects generally involve numerous objects to be lifted by a crane in congested areas. Previous research studies in industrial construction have focused on: (1) identifying feasible crane lift methods such as the pick from fixed position (PFP) and pick and walking operation (PWO); and (2) planning detailed motions of mobile crane operation using 3D visualization. However, this system lacks decision making tools by which to select the best crane lift path in terms of high crane performance (productivity) when lifted objects have more than one possible crane lift path. To overcome this deficiency, this paper has presented a system integrating $3 \mathrm{D}$ visualization and simulation, known as automated post-3D visualization simulation. The 
purpose of this system is to select the best crane operation by analyzing crane performance in the simulation based on the $3 \mathrm{D}$ visualization of crane operations. This system has been successfully implemented in a case study by representing the expected cycle times of crane operations for the lifted object. We are presently expanding the work scope such that the future system will be able to apply this system to plan an optimal material handling system, as well as project schedules to increase project productivity.

\section{Acknowledgements}

The authors wish to acknowledge the financial support of the Natural Sciences and Engineering Research Council of Canada (NSERC) Collaborative Research and Development (CRD) program. The participation and support of our collaboration partner for this research, PCL Industrial Management Inc., is also appreciated.

Table 2. An example of the information for simulation

\begin{tabular}{rrrrrrrrrr}
\hline ObjID & TrackingNo & \multicolumn{1}{l}{ Weight } & \multicolumn{1}{l}{ Radius } & Capacity & \multicolumn{1}{l}{ Factor } & \multicolumn{1}{l}{ LiftAngle } & LiftHeight & Speed & MotionID \\
\hline \hline 103 & 12049 & 54762 & 144.1074524 & 286600 & 19.10746685 & -71.99176025 & 0 & 131.9396655 \\
103 & 12049 & 54762 & 144.1074524 & 286600 & 19.10746685 & 81.1828537 & 0 & 131.9396655 & 2 \\
103 & 12049 & 54762 & 144.1074524 & 286600 & 19.10746685 & 6.895394825 & 0 & 131.9396655 \\
103 & 12049 & 54762 & 144.1074524 & 286600 & 19.10746685 & 98.82134247 & 0 & 131.9396655 \\
103 & 12049 & 54762 & 144.0997314 & 286600 & 19.10746685 & 0 & 53.67518997 & 206.2866199 \\
103 & 12049 & 314952 & 144.0997314 & 286600 & 109.8925331 & 0 & 7 & 63.20936399 \\
103 & 12049 & 314952 & 141.6588593 & 286600 & 109.8925331 & -143.8604584 & 0 & 40.42832418 \\
103 & 12049 & 314952 & 141.6588593 & 286600 & 109.8925331 & -108.4297333 & 0 & 40.42832418 & 7 \\
103 & 12049 & 314952 & 141.6588593 & 286600 & 109.8925331 & 0 & 0 & 40.42832418 \\
103 & 12049 & 314952 & 141.6588593 & 286600 & 109.8925331 & 0 & 0 & 40.42832418 \\
103 & 12049 & 314952 & 141.6588593 & 286600 & 109.8925331 & 0 & 4 & 7 \\
103 & 12049 & 314952 & 141.6588593 & 286600 & 109.8925331 & 0.42832418 & 7 \\
103 & 12049 & 314952 & 141.6588593 & 286600 & 109.8925331 & 0 & 0 & 40.42832418 & 7 \\
103 & 12049 & 314952 & 141.516449 & 286600 & 109.8925331 & 78.28238054 & 0 & 40.42832418 & 7 \\
103 & 12049 & 314952 & 141.6588593 & 286600 & 109.8925331 & 22.46762276 & 0 & 40.42832418 & 8 \\
103 & 12049 & 314952 & 141.6588593 & 286600 & 109.8925331 & 0 & 40.42832418 & 8 \\
103 & 12049 & 314952 & 141.6588593 & 286600 & 109.8925331 & 0 & 0 & 63.20936399 & 15 \\
& & & & & & 0 & 0 & 40.42832418
\end{tabular}

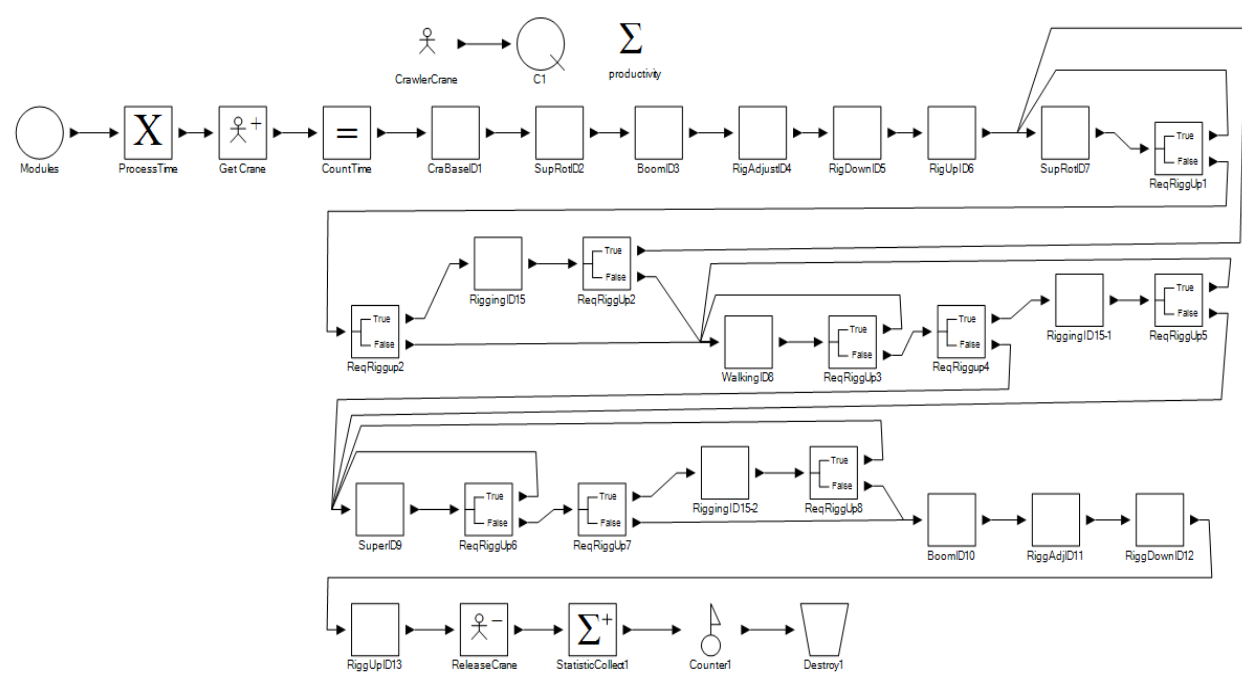

Figure 5. A simulation model of mobile crane operation in Simphony.NET 4 


\section{References}

[1] AlBahnassi, H. and Hammad, A. Near real-time motion planning and simulation of cranes in construction: Framework and system architecture. Journal of Computing in Civil Engineering, 26(1): 54-63, 2012.

[2] Al-Hussein, M., Niazm, M. A., Yu, H., and Kim, $\mathrm{H}$. Integrating $3 \mathrm{D}$ visualization and simulation for tower crane operations on construction sites. Automation in Construction, 15: 554-562, 2006.

[3] Ali, M. S. A. D., Babu, N. R., and Varghese, K. Collision free path planning of cooperative crane manipulators using genetic algorithm. Journal of Computing in Civil Engineering, 19(2): 182-193, 2005.

[4] Al-Hussein, M., Alkass, S., and Moselhi, O. Optimization algorithm for selection and on-site location of mobile cranes. Journal of Construction Engineering and Management, 131(5): 579-590, 2005.

[5] Akinci, B., Fischer, M., and Kunz, J. Automated generation of work spaces required by construction activities. Journal of Construction Engineering and Management, 128(4): 306-315, 2002.

[6] AbouRizk, S. and Mohamed, Y. Optimal construction project planning. Proc., Winter Simulation Conf., Vol. 2, 1704-1708.

[7] Chang, Y. C., Hung, W. H., and Kang, S. C. A fast path planning method for single and dual crane erections. Automation in Construction, 22: 468-480, 2012.

[8] Han, S. H., Lei, Z., Bouferguène, A., Al-Hussein, M., and Hermann, U. Automated motion planning of mobile crane operation in congested industrial projects using 3D visualization: rotation analysis. Journal of Computing in Civil Engineering, (submitted Jan., 2014).

[9] Han, S. H., Hasan, S., Bouferguène, A., AlHussein, M., and Kosa, J. Utilization of 3D visualization of mobile crane operations for modular construction on-site assembly: A case study implementation. Accepted (Apr., 2014) to Journal of Management in Engineering.

[10] Han, S. H., Al-Hussein, M., Al-Jibouri, S., and $\mathrm{Yu}, \mathrm{H}$. Automated post-simulation visualization of modular building production assembly line. Automation in Construction, 21: 229-236, 2012.

[11] Hasan, S., Bouferguène, A., Al-Hussein, M., Gillis, P., and Telyas, A. Productivity and $\mathrm{CO}_{2}$ emission analysis for tower crane utilization on high-rise building projects. Automation in
Construction, 31: 255-264, 2013.

[12] Hasan, S., Al-Hussein, M., Hermann, U. H., and Safouhi, $H$. Interactive and dynamic integrated module for mobile cranes supporting system Design. Journal of Construction Engineering and Management, 136(2): 179-186, 2010.

[13] Huang, C., Wong, C. K., and Tam, C. M. Optimization of tower crane and material supply locations in a high-rise building site by mixedinteger linear programming. Automation in Construction, 20(5): 571-580, 2011.

[14] Kang, S. C., Chi, H. L., and Miranda, E. Threedimensional simulation and visualization of crane assisted construction erection processes. Journal of Computing in Civil Engineering, 23(6): 363-371, 2009.

[15] Lei, Z., Taghaddos, H., Hermann, U., and AlHussein, M. A methodology of mobile crane lift path checking in heavy industrial projects. Automation in Construction, 31: 41-53, 2013.

[16] Li, Y. and Liu, C. Integrating field data and 3D simulation for tower crane activity monitoring and alarming. Automation in Construction, 27:111-119, 2012.

[17] Lin, Y., Wu, D., Wang, X., Wang, X., and Gao, S. Statics-based simulation approach for twocrane lift. Journal of Construction Engineering and Management, 138(10): 1139-1149, 2012.

[18] Lai, K. C. and Kang, S. C. Collision detection strategies for virtual construction simulation. Automation in Construction, 18: 724-736, 2009.

[19] Olearczyk, J., Bouferguène, A., Al-Hussein, M., and Hermann, R. Crane lifted object spatial trajectory automation analysis. Automation in Construction, (Nov., 2012).

[20] Park, T., Kim, M., Kim, C., and Kim, H. Interactive $3 \mathrm{D}$ CAD for effective derrick crane operation in a cable-stayed bridge construction. Journal of Construction Engineering and Management, 135(11): 1261-1270, 2009.

[21] Safouhi, H., Mouattamid, M., Hermman, U., and Hendi, A. An algorithm for the calculation of feasible mobile crane position areas. Automation in Construction, 20(4): 360-367, 2011.

[22] Sawhney, A. and Mund, A. Adaptive probabilistic neural network-based crane type selection system. Journal of Construction Engineering and Management, 128(3): 265273, 2002.

[23] Sivakumar, P. L., Varghese, K., and Babu, N. R. Automated path planning of cooperative crane lifts using heuristic search. Journal of 
Computing in Civil Engineering, 17(3): 197-207, 2003.

[24] Tantisevi, K. and Akinci, B. Simulation-based identification of possible locations for mobile cranes on construction sites. Journal of Computing in Civil Engineering, 22(1): 21-30, 2003.

[25] Wu, D., Lin, Y., Wang, X., Wang, X., and Gao,
S. Algorithm of crane selection for heavy lifts. Journal of Computing in Civil Engineering, 25(1): 57-65, 2011.

[26] Zhang, C. and Hammad, A. Improving lifting motion planning and re-planning of cranes with consideration for safety and efficiency. Advanced Engineering Informatics, 26: 396-410, 2012. 